\title{
PORTAL UTILIDADES - UMA FERRAMENTA PARA GESTÃO OPERACIONAL USANDO O PIMS NA TKCSA*
}

Claudio José Martins da Silva ${ }^{1}$ Elio Souza Portes ${ }^{2}$ Alessandro Andrade Maia ${ }^{3}$

\section{Resumo}

A ferramenta PIMS (Plant Information Management System) possibilita, através dos recursos de conexão com o sistema de automação, a qualquer usuário da rede coorporativa da TKCSA o acesso à base de dados do processo produtivo e, através de suas funcionalidades o desenvolvimento, sem restrições, análises "on line" da planta banco a partir dos bancos de dados histórico que ele possui. Este trabalho apresenta a experiência da gerência técnica de utilidades da TKCSA no desenvolvimento de ferramenta de supervisão e gestão de informações através da ferramenta PIMS, intitulada Portal Utilidades. Com a ferramenta desenvolvida 0 corpo técnico é capaz de realizar análises on line ou consultando a base de dados do sistema, gerando diagnósticos, recomendações ou mesmo orientações às equipes de operação sobre o desempenho do sistema de Utilidades. Além de permitir todos os tipos de usuários (supervisores, mantenedores, operadores e outros) a consulta das informações a qualquer tempo suportando em atividades, como por exemplo, planejamento de manutenção e manobras dentre outras consultas.

Palavras-chave: PIMS; Supervisão; Automação; Portal de informações.

\section{UTILITIES PORTAL - A TOOL FOR OPERATIONAL MANAGEMENT USING THE PIMS IN TKCSA}

\section{Abstract}

The tool PIMS (Plant Information Management System) enables, through the connection resources with the automation system, any user of the CSA corporate network access to the database and, through its functionalities development without restriction on line analysis of the plant from the historical database. This paper presents the experience of the Technical Utilities Department of CSA utilities in the development of supervisory tools and information management through the PIMS tool, at mean, Utilities Portal. With the Utilities Portal developed the staff is able to perform analyzes on line or by consulting the system database, generating diagnoses, recommendations or guidelines for operations teams on the performance of Utilities system. In addition to allowing all types of users (supervisors, maintainers, operators and other) consultation of information at any time supporting activities, such as maintenance planning and maneuvering among other queries.

Keywords: PIMS; Supervision; Automation; Information portal.

Engenheiro Especialista de Manutenção, TKCSA, Rio de Janeiro, RJ, Brasil; claudio.martins@thyssenkrupp.com.

Engenheiro de Automação, TKCSA, Rio de Janeiro, RJ, Brasil; elio.portes @thyssenkrupp.com

Engenheiro Mecânico, ex-funcionário da TKCSA, Fortaleza, CE, Brasil. 


\section{INTRODUÇÃO}

O sistema de armazenagem e gerenciamento de dados está cada vez mais presentes especialmente nas plantas mais recentes e possuam uma arquitetura de automação que trata os dados desde o chão de fábrica (instrumentos, medidores, equipamentos, etc.) até os sistemas de gerenciamento de produção, planejamento e financeiro. Dentro desta arquitetura de automação o PIMS (Plant Information Management System), exerce papel fundamental como elo de ligação entre os controladores de processo e sistemas SCADA, coletando, armazenando e disponibilizando para todos os usuários a base de dados históricos.

Os dados históricos armazenados no PIMS permitem diversas formas de manuseio dos dados, permitindo às equipes de gestores, engenheiros, técnicos e operadores o gerenciamento de informações e tomadas de decisão baseada em dados reais e atualizadas dos processos.

O presente trabalho apresenta a ferramenta desenvolvida pela equipe da gerência técnica de utilidades da TKCSA a partir das funcionalidades do software do PIMS que foi intitulada Portal Utilidades e, que possui as funções de gerenciamento "on line" permite aos usuários do processo e componentes da planta de utilidades, bem como um browser (Navegador) funcionando como porta de entrada a outras informações de interesse para a gestão de informações.

\subsection{PIMS na Arquitetura de Automação}

O PIMS nasceu na indústria de processos contínuos mais propriamente na indústria química e petroquímica para resolver o problema da fragmentação de dados e proporcionar uma visão unificada do processo. Numa primeira fase, o PIMS passa a se constituir na ferramenta fundamental para o engenheiro de processos. A partir de uma estação ele pode visualizar tanto os dados de tempo real como históricos da planta. Pode montra tabelas, gráficos de tendência e sinópticos e eliminar as ilhas de informação, concentrando em uma única base de dados informação sobre todos os aspectos de uma planta. A implantação de um PIMS facilita a implantação de outros módulos de software como reconciliador de dados, sistema especialista, Supply Chain Manager e facilita a integração de sistemas ERP com o chão de fábrica. A capacidade de gerar outros dados através de cálculos e de armazená-los por longos períodos de tempo sem ter que enviá-los a um mainframe constitui um grande ganho para o analista de processos. Ele deixa de depender do pessoal do departamento de informática e passa a gerar relatórios sem ter que se preocupar se o dado se origina num CLP, num sistema SCADA ou SDCD.

A infraestrutura de automação de PIMS possui os seguintes componentes:

a) Servidor Principal;

b) Servidor de comunicação;

c) Estações Clientes;

d) Banco de dados relacional;

e) Infraestrutura de rede (coorporativa e automação). 


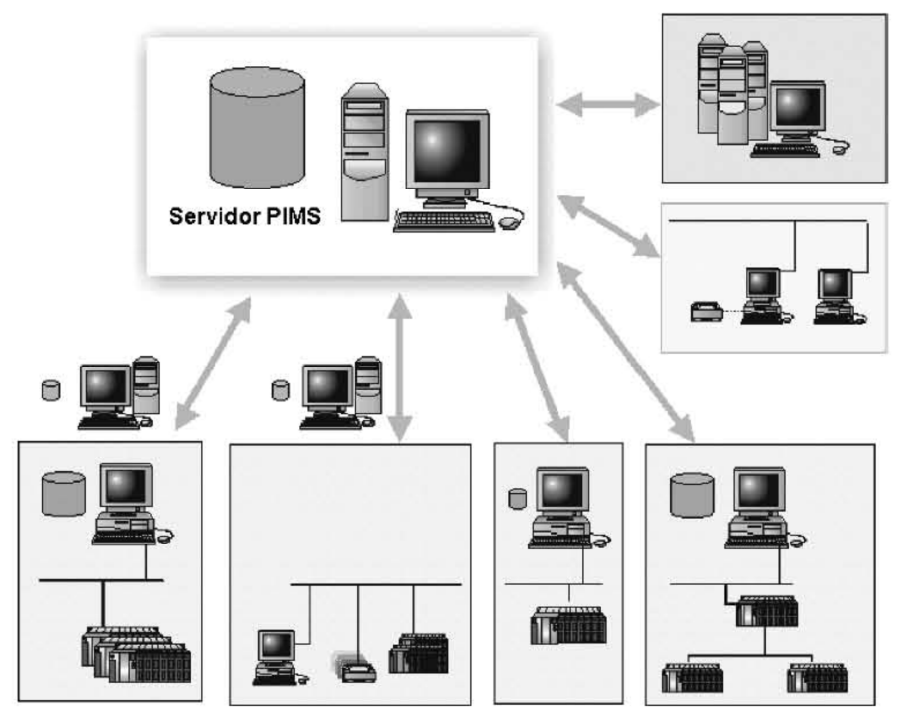

Figura 1: Infraestrutura de automação do sistema PIMS [6].

\subsection{Arquitetura de Automação na TKCSA}

O complexo siderúrgico da TKCSA possui uma estrutura em níveis e com as seguintes funções e objetivos.

Tabela 01: Abrangência dos níveis de hierarquia de automação [2]

\begin{tabular}{|c|c|}
\hline Nível & Componentes e funções \\
\hline $\begin{array}{l}\text { Nível } 0 \text { - Nível de } \\
\text { campo }\end{array}$ & $\begin{array}{l}\text { Sensores-atuadores } \\
\text { Todos equipamento de campo da automação de processos são colocados } \\
\text { neste nível. }\end{array}$ \\
\hline $\begin{array}{l}\text { Nível } 1 \text { - Nível de } \\
\text { gerenciamento de } \\
\text { processo }\end{array}$ & $\begin{array}{l}\text { Este constitui o núcleo de automação e também é dividida em duas } \\
\text { camadas, que são: } \\
\text { Relacionadas com o campo } \\
\text { - Medição } \\
\text { - Malha de controle Controle } \\
\quad \text { Open- loop } \\
\quad \text { Closed-loop } \\
\text { - Monitoração } \\
\text { - Segurança } \\
\text { Controle e gerenciamento de processo } \\
\text { - Supervisão } \\
\text { - Operação } \\
\text { - Configuração } \\
\text { - } \quad \text { Gerametrização } \\
\end{array}$ \\
\hline $\begin{array}{l}\text { Nivel2 - Nível de } \\
\text { planta }\end{array}$ & $\begin{array}{l}\text { Nível superior em uma determinada planta (setor) } \\
\text { - } \quad \text { Gentrole de produção } \\
\text { - Análise de processo manutenção } \\
\text { - Modelagem e simulações } \\
\text { - Relatórios }\end{array}$ \\
\hline $\begin{array}{l}\text { Nível 3 - Nível } \\
\text { Empresa }\end{array}$ & $\begin{array}{l}\text { Gerenciamento de todas Instalações da toda a empresa como: } \\
\text { Planejamento de produção, gestão de Controle, Gestão de Informação, } \\
\text { Vendas e Suprimentos, Gestão de Pessoas e outros são colocados neste } \\
\text { nível }\end{array}$ \\
\hline
\end{tabular}




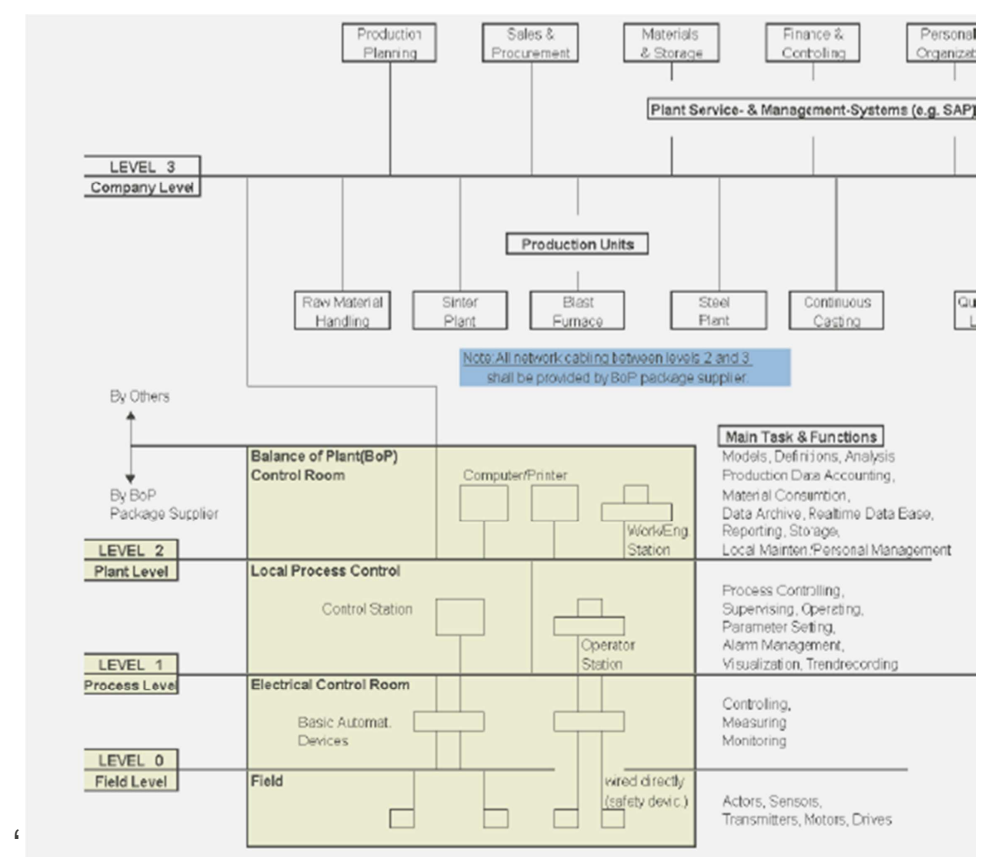

Figura 2: Ilustração da infraestrutura de automação da TKCSA [3,5].

\subsection{PIMS da TKCSA}

\subsubsection{Infraestrutura:}

A infraestrutura do PIMS da TKCSA é composta de:

\subsubsection{Funcionalidades}

As principais funcionalidades do PIMS são:

a) Comunicação com os diversos sistemas de gerenciamento de processo das plantas

b) Armazenagem de dados para utilização em análises e gerenciamento de informações (gráficos de tendências, dados estatísticos, dados atuais, outros).

c) Interação com o nível superior de automação (Planejamento de produção, controle de produção-MES e ERP- SAP).

\section{MATERIAIS E MÉTODOS}

\subsection{Portal Utilidades}

O Portal Utilidades se apresenta como uma ferramenta integradora de informações, técnicas e gerenciais que disponibiliza ao usuário um acesso rápido a todo tipo de informação pertinente às necessidades do usuário.

Podemos dividir o portal em duas funções complementares gerenciamentos de dados de processo via PIMS e Browser de Navegação que permite acesso a informações e que se complementam e oferecem ao usuário uma ferramenta de gestão e acesso a informações.

O usuário pode ter certeza que as informações geradas são de qualidade e representam a realidade operacional.

A tela principal do portal é o único ponto de acesso às informações onde toda planta de utilidades pode ser consultada. 


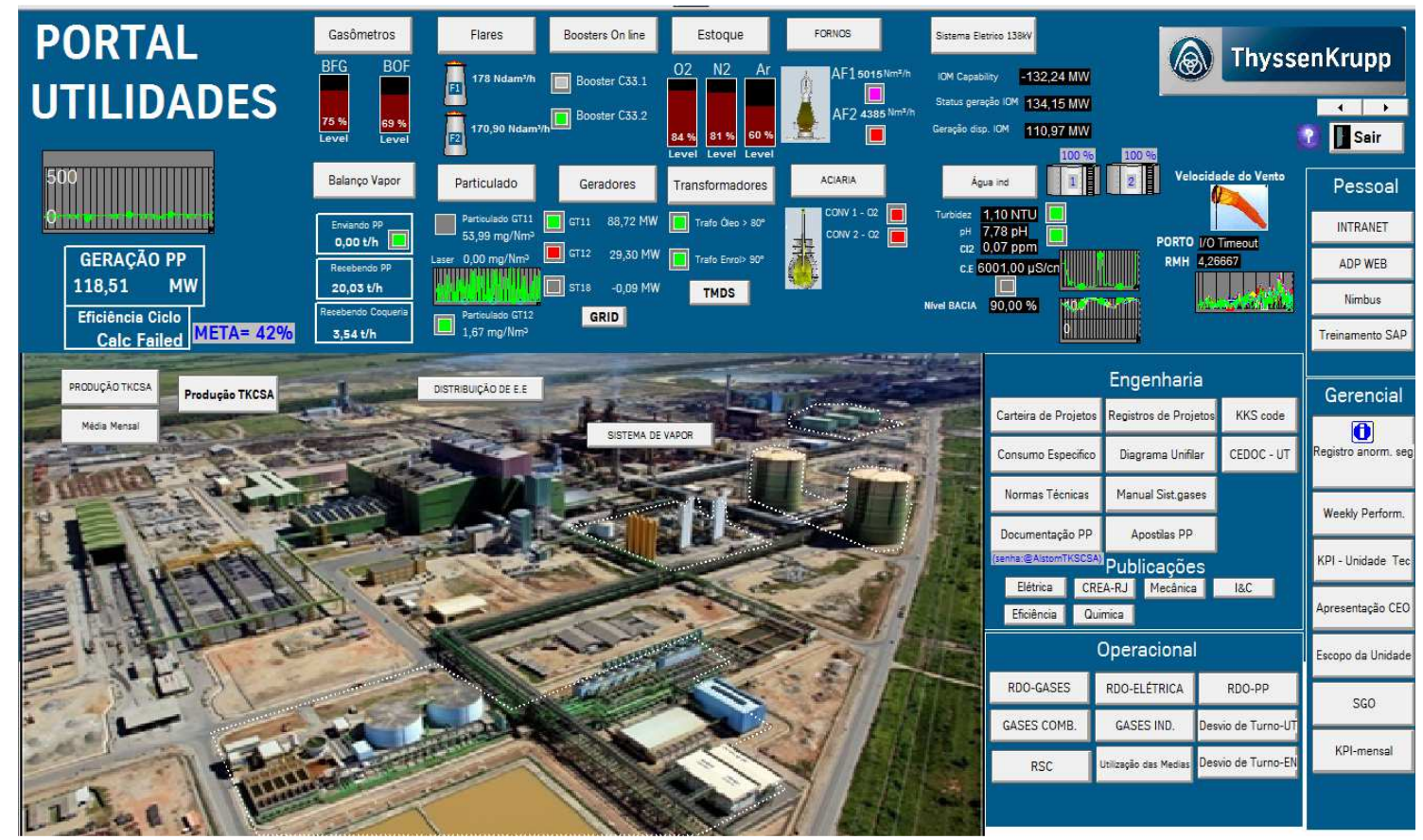

Figura 3: Tela principal do portal Utilidades

\subsubsection{Funcionalidades via PIMS}

Com apenas um clique na foto, operação das áreas de água, gases técnicos, sistemas de gases, geração de gases e energia elétrica e finalmente a distribuição de energia são monitoradas 24 horas, proporcionando identificar oportunidades para alcançar melhores resultados.

\subsubsection{Gráfico de tendências e tempo real}

Com essa funcionalidade o Usuário é capaz de acompanha o perfil da grandeza monitorada em um período definido e o valor instantâneo da variável monitorada.

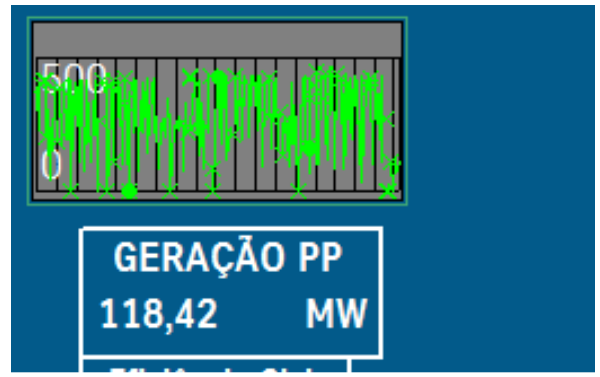

Figura 4: Gráfico de Tendência de variável monitorada

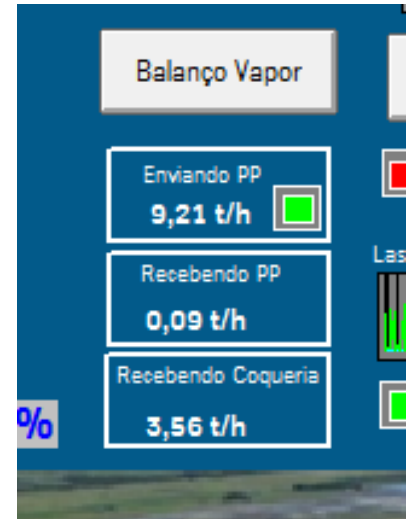

Figura 5: Valor instantâneo de variável monitorada 


\subsubsection{Controle de nível de tanques}

Com essa funcionalidade o usuário é capaz verificar o valor instantâneo do nível do tanque monitorado e ilustra o componente através da utilização do recurso biblioteca de símbolos (Symblos Library).
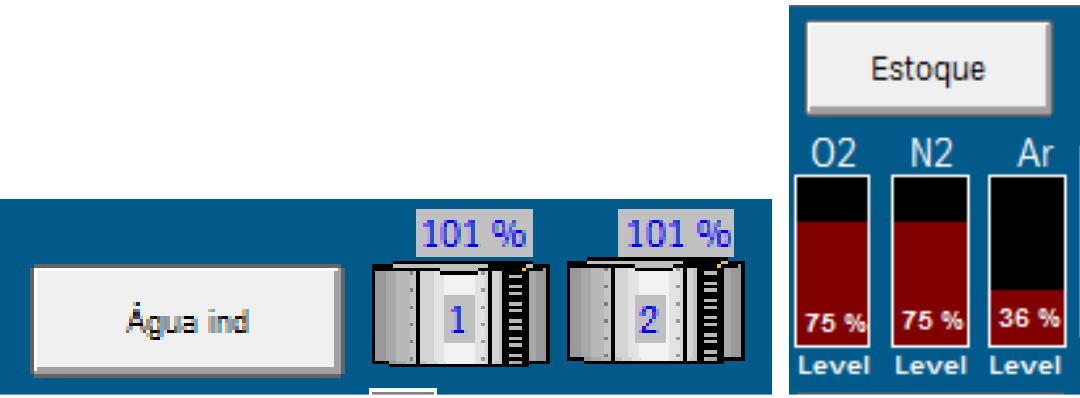

Figura 6: Valor instantâneo dos tanques de água tratada e criogênicos

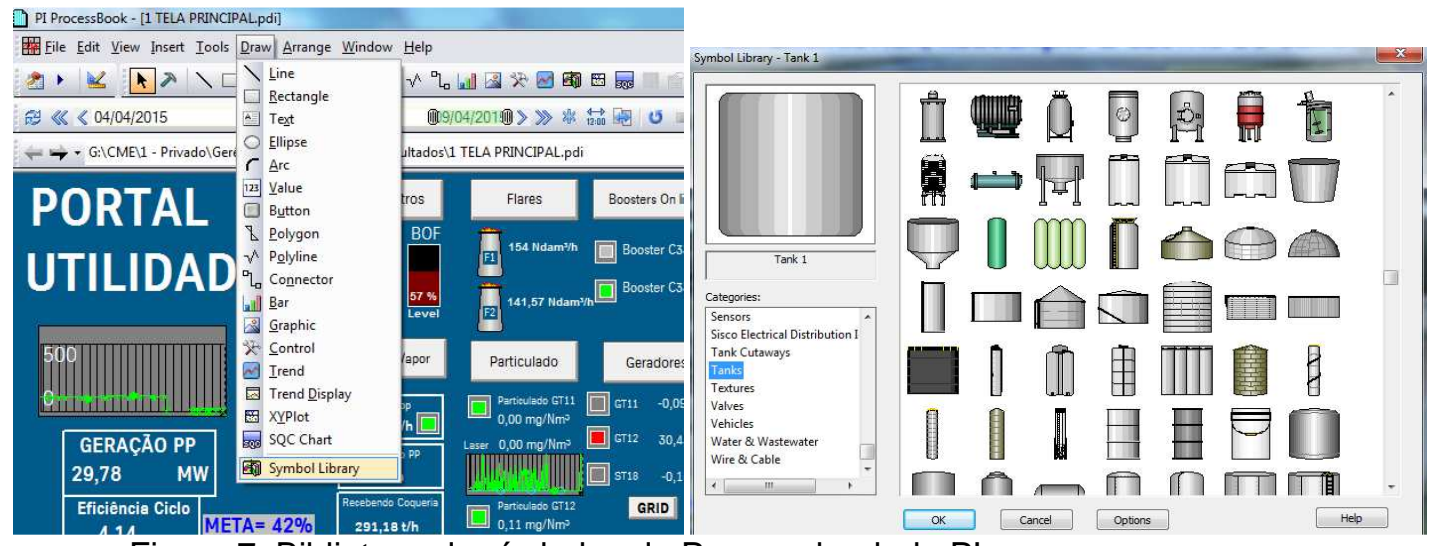

Figura 7: Biblioteca de símbolos do Process book do PI

\subsubsection{Queima nos Flares}

Com essa funcionalidade o usuário é capaz verificar o valor instantâneo de queima de gás de alto forno. Adicionalmente através da funcionalidade de botões acessa-se o gráfico de tendência para análise de comportamento da queima ao longo de um período.
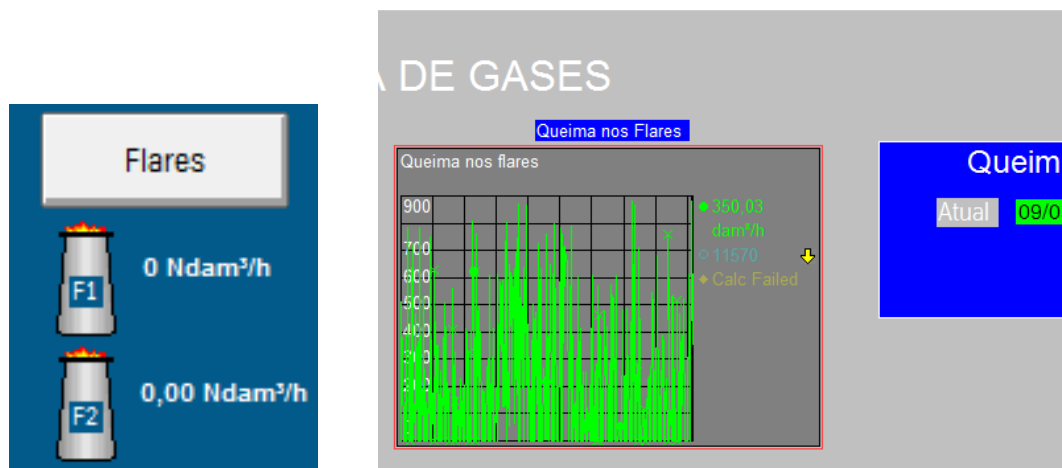

Figura 8: Valor instantâneo de queima de BFG nos flares e gráfico de tendência

\subsubsection{Performance dos gasômetros}

Equipamentos de suma importância no sistema de controle e distribuição de gases, os gasômetros podem ter sua performance supervisionada através do controle dos afastamento do costado "clearance". Com esse recurso o especialista do sistema de gás pode propor inspeções mais detalhadas ou mesmo adiar inspeções no interior dos gasômetros. 


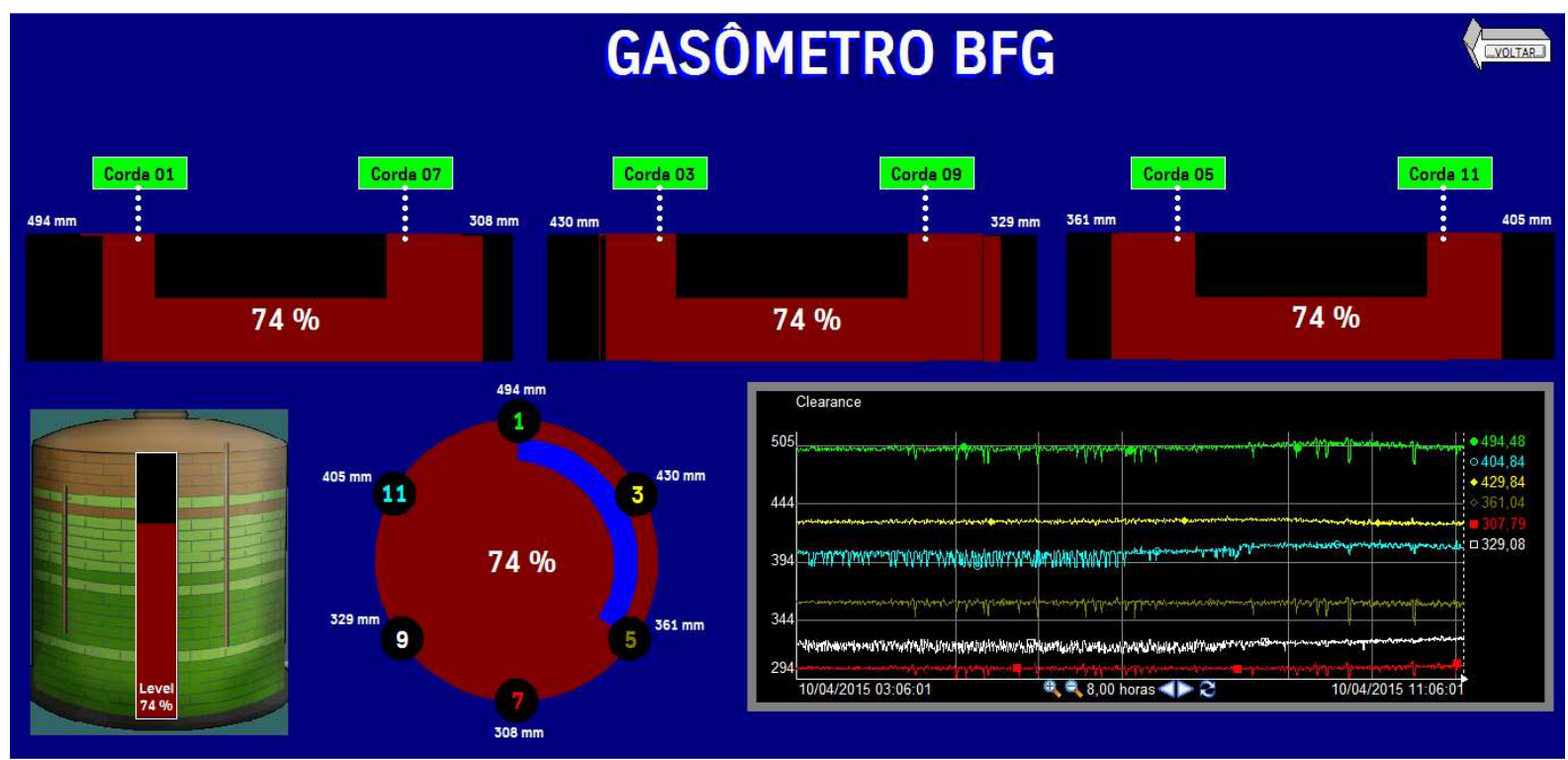

Figura 9: Performance do gasômetro

\subsubsection{Temperatura de transformadores}

Com essa funcionalidade o usuário é capaz constatar através da indicação "VERDE" ou "VERMELHA" se todos os transformadores estão com a temperatura de óleo ou enrolamento acima dos valores de alarme.

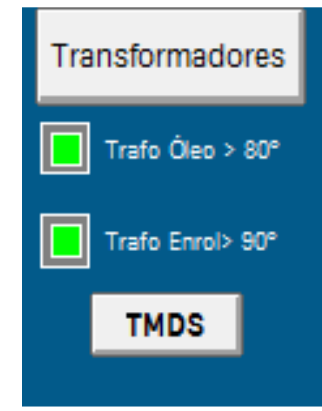

Figura 10: Indicativo de existência de transformadores acima da temperatura de alarme

\subsubsection{Alarme ultrapassagem de valores máximos}

Com essa funcionalidade o usuário é capaz constatar através da indicação "VERDE" AMARELA ou "VERMELHA" se os valores medidos estão dentro da normalidade, situações de alarme ou situação emergencial.

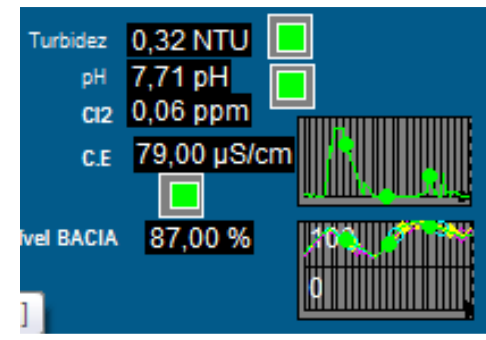

Figura 11: Indicativo de situação NORMAL no valor de condutividade da água de captação 
FORNOS

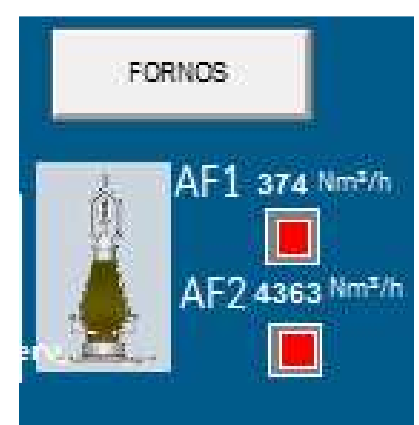

Figura 12: Indicativo de situação EMERGENCIAL de operação dos fornos

\subsubsection{Acesso a análises específicas de áreas}

Com essa funcionalidade o usuário é capaz de acessar através o recurso de botões uma tela específica de análise equipamento crítico no processo. Por exemplo os booster de gás de aciaria, realizando análise perfis de temperatura ou variação da vibração do sistema.

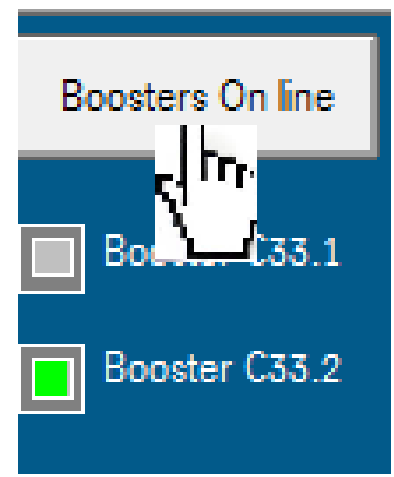

Figura 13: Indicativo de situação EMERGENCIAL de operação dos fornos

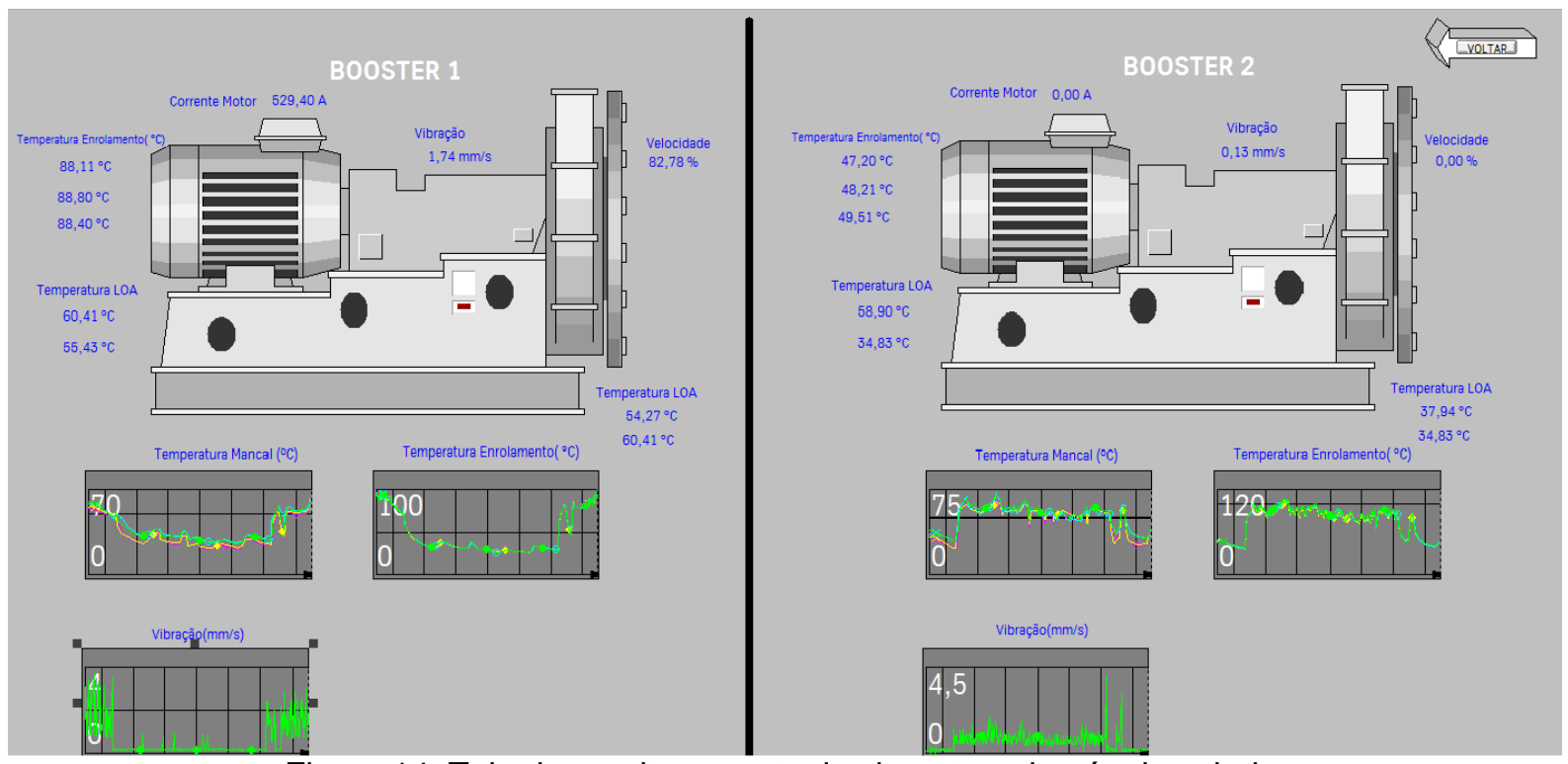

Figura 14: Tela de monitoramento dos boosters de gás de aciaria

\subsubsection{Gerenciamento da distribuição de consumo de utilidades}

O gerenciamento de consumos permite ao gestor ou ao analista uma visão geral do consumo instantâneo de uma determinada Utilidade. 


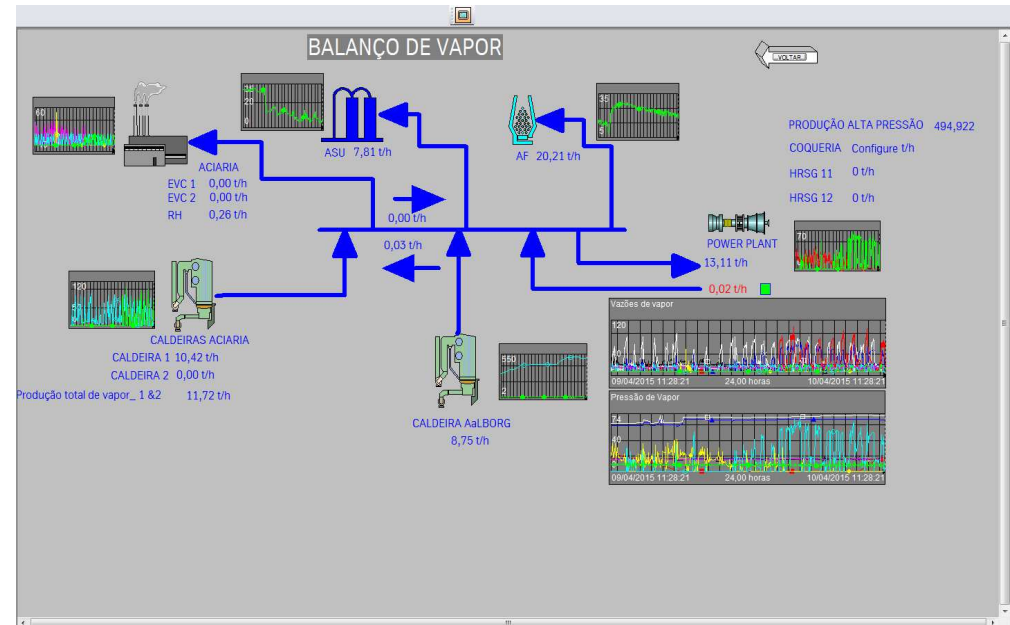

Figura 15: Distribuição da distribuição do consumo de Vapor

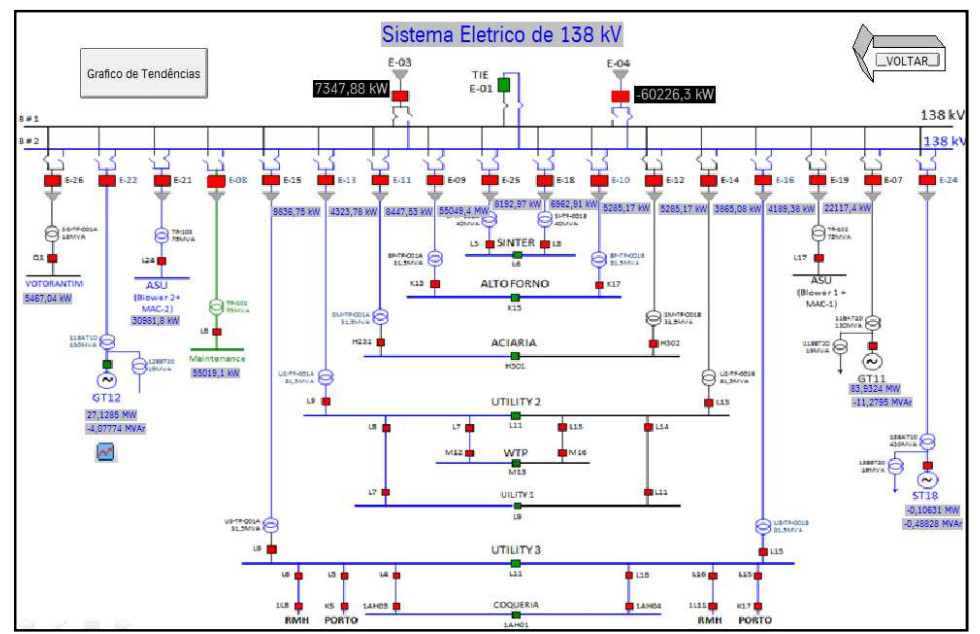

Figura 16: Distribuição da distribuição do consumo de Energia Elétrica

\subsubsection{Funcionalidades como Browser (navegador)}

Documentações e informações técnicas podem ser localizadas em ENGENHARIA. Em OPERACIONAL monitoramos o desempenho operacional da área de utilidades, acessando relatórios e apresentações.

Sem sair do Portal Utilidades podemos acessar alguns recursos de $\mathrm{RH}$, através do PESSOAL.

A utilização do sistema PIMS como gerenciador das interfaces com a origem dos dados técnicos e informações de processos dão credibilidade ao conteúdo disponível.

O recurso "botão" permite a criação de um link através de um endereço específico durante o processo de parametrização do botão.

Por exemplo, o acesso ao banco de dados de normas técnicas da TKCSA através do botão NORMAS TÉCNICAS e diretamente através do botão SGO acessar o sistema de gerenciamento operacional da gerência de geração de energia. 


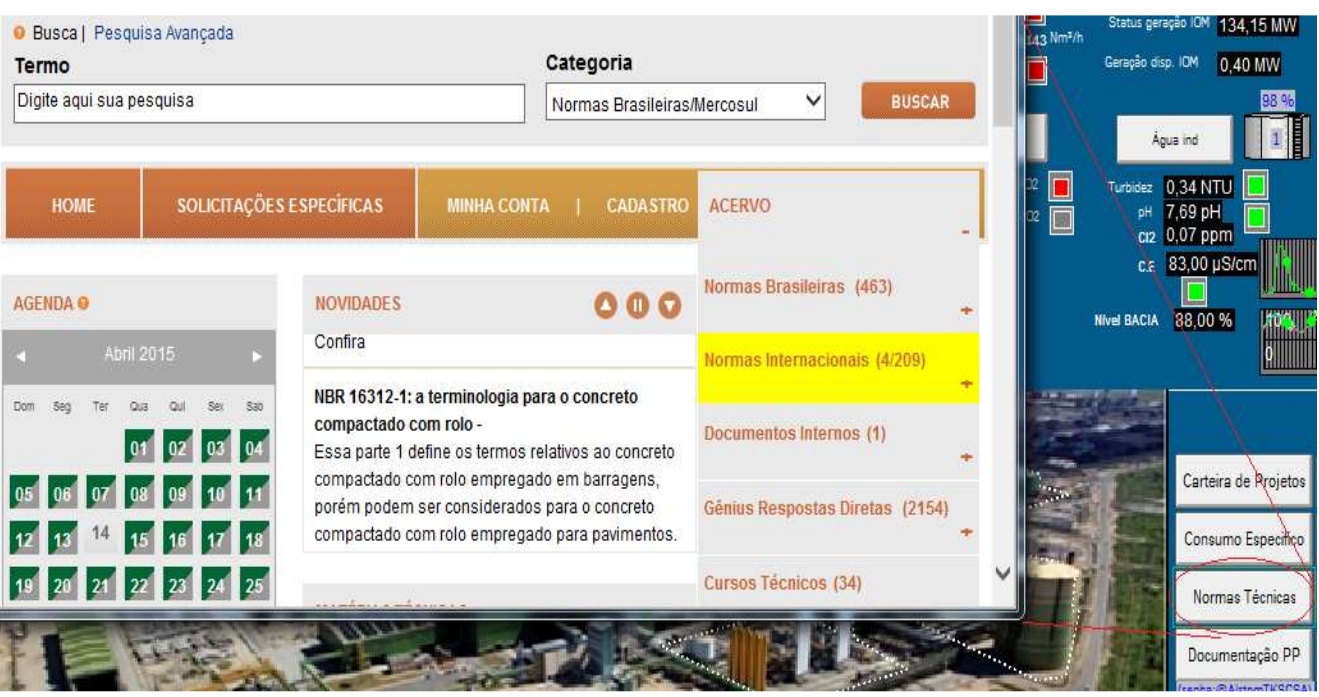

Figura 17: Funcionamento "browse" acessando outros ambientes - GED WEb

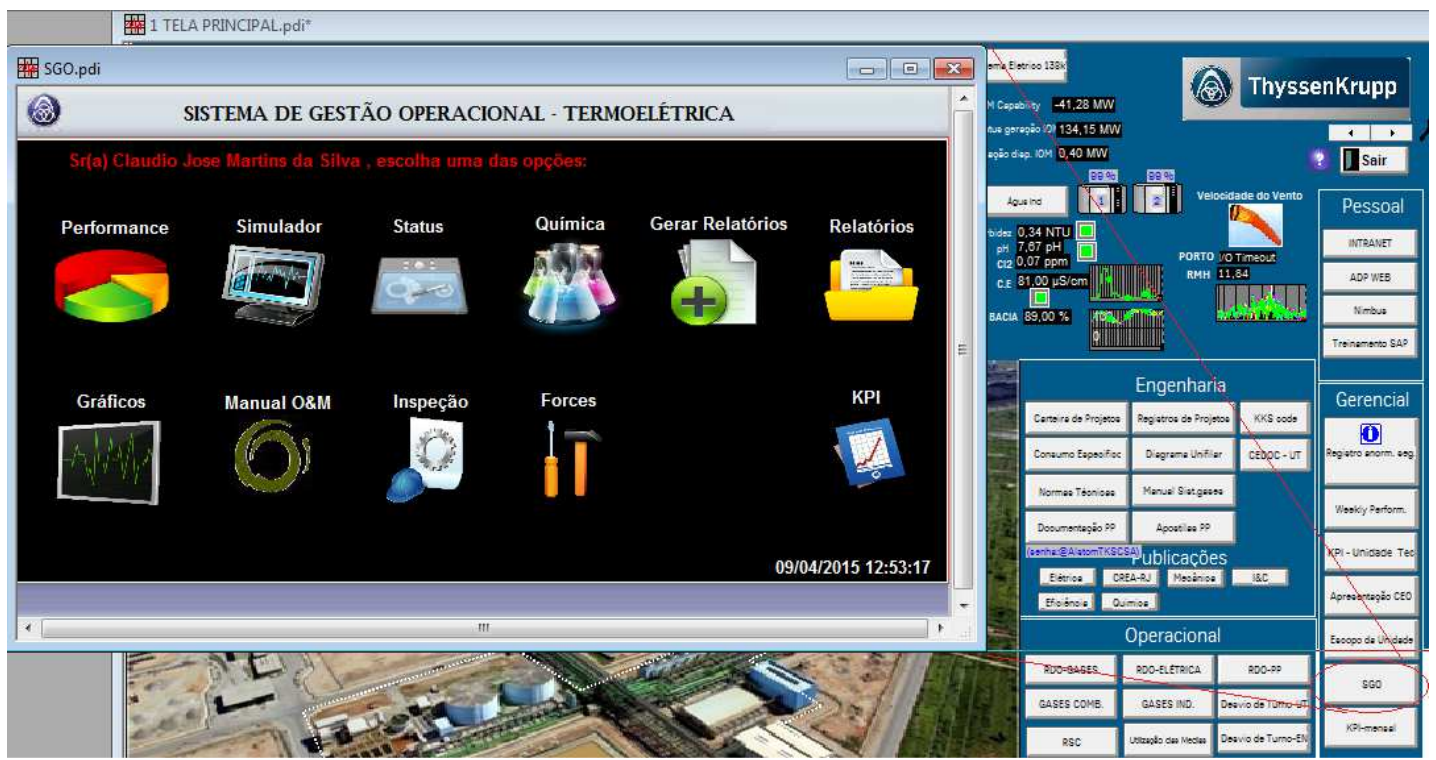

Figura 18: Funcionamento "browse" acessando outros ambientes - GED WEb e SGO

\section{RESULTADOS E DISCUSSÃo}

\subsection{Resultados Tangíveis}

Apesar da função de controle e supervisão seja da responsabilidade do sistema de automação e controle da planta, com a utilização do Portal Utilidades possibilitou através de uma gestão técnica adicional, o maior conhecimento do perfil de variação do Clearance dos gasômetros através dos gráficos de tendência e atuar solicitando intervenção de correção no desequilíbrio antes das diferenças cresçam a valores que represente risco a operação do gasômetro.

Mesmo como uma ferramenta de gestão da base de dados a utilização do portal Utilidades já possibilitou diversas análises de desempenho como: perfil de aumento de vibração do booster de gas BOF, aumento da temperatura dos transformadores de alta tensão e balanço de vapor. 


\subsection{Resultados Intangíveis}

Os resultados alcançados com a utilização do Portal foram especialmente percebidos pelos coordenadores de operação e manutenção que, com uma rápida olhada na tela principal do Portal Utilidades pode verificar as condições do processo do fornecimento de gases, água, criogênicos, vapor e energia elétrica.

Toda a equipe técnica da gerencia técnica de utilidades conseguiu sugerir algumas funcionalidades novas ao longo do tempo de utilização do Portal que implementadas proporcionou mais interesse na utilização da ferramenta com propósitos específicos a sua área de atuação.

O ponto mais relevante do desenvolvimento e utilização de ferramentas como ao Portal Utilidades é a universalização da informação para todos os níveis da cadeia de produção. Com o acesso ao banco de dados de forma amigável e rápida entre homem e a máquina podemos dizer que realmente entramos em uma nova era na gestão como propôs, Silva [1].

\subsection{Discussão}

A exploração aprofundada das funcionalidades do PIMS apresentadas por Carvalho [6] e Rabelo [4] e organizando através de uma interface homem- máquina como a proposta pelo Portal Utilidades possibilitou a criação de uma ferramenta de gestão poderosa para os gestores operacionais e de manutenção.

\section{CONCLUSÃO}

O Portal utilidades como ferramenta de suporte ao gestor operacional e ao engenheiro de processo proporciona alternativas de análise e de tomada de decisão que permitindo a antecipação de situações críticas, não somente na gestão do tempo real, como também que indicam ações estratégicas a serem tomadas.

O desenvolvimento da ferramenta teve também um importante papel no para outros usuários que conseguiram a partir da contribuição com o desenvolvimento outras funcionalidades, uma maior interação com o PIMS e aumentando o nível de utilização software.

A única certeza que chegamos com o desenvolvimento do Portal é que ele tem vida, esta em constante atualização de suas funcionalidades e, que a utilização por um maior numero de usuários é que vai elevar cada vez mais o seu nível de importância como uma ferramenta de gestão operacional e técnica.

\section{REFERÊNCIAS}

1 Silva, FJ, Pinto MM, Guimarães F M - Nova era para otimização do sistema de gestão operacional na usina termelétrica Thyssenkrupp CSA siderúrgica do atlântico - 34

Seminário de Balanços Energéticos Globais e Utilidades e $28^{\circ}$ Encontro de Produtores e Consumidores de Gases Industriais, 28 a 30 de agosto de 2013, Vitória, ES.

2 Matéria publicada na revista Mecatrônica Atual; Ano: 10; N 54; Jan / Fev - 2012.

3 Alessandro J. Souza AAJ., Guedes A. - Notas de aula : Gerência da Informação nos Processos de Automação Industrial Programa de Pós-Graduação em Engenharia Elétrica Redes Industriais.

4 Rabelo RJ.Notas de aula: Integração de Sistemas Corporativos- UFSC.

5 Seixas. PIMS - Process Information Management System - Uma introdução- Cap. 6, UFMG; 2003.

6 Carvalho FB, Torres FB, Fonseca MO, Filho CS. Sistema PIMS conceituação Uso e Benefícios - Revista Tecnologia em Metalurgia e Materiais. 2005;1(4):1-5. 